\title{
Fauna de aranhas (Arachnida, Araneae) em diferentes estágios do cultivo do arroz irrigado em Cachoeirinha, RS, Brasil
}

\author{
Everton N. L. Rodrigues ${ }^{1,3}$, Milton de S. Mendonça Jr. ${ }^{1,2} \&$ Ricardo Ott $^{3}$
}

1. Programa de Pós-Graduação em Biologia Animal, Instituto de Biociências, Universidade Federal do Rio Grande do Sul. Bolsista CNPq. Av. Bento Gonçalves, 9500, Bloco IV, Prédio 43435, 91501-970 Porto Alegre, RS. (enlrodrigues@ yahoo.com.br)

2. Departamento de Ecologia, Instituto de Biociências, Universidade Federal do Rio Grande do Sul. Av. Bento Gonçalves, 9500, Bloco IV, Prédio 43422, 91501-970 Porto Alegre, RS. (milton.mendonca@ufrgs.br)

3. Museu de Ciências Naturais, Fundação Zoobotânica do Rio Grande do Sul. Rua Dr. Salvador França, 1427, 90690-000 Porto Alegre, RS. (rott@fzb.rs.gov.br)

\begin{abstract}
Spider fauna (Arachnida, Araneae) in differents stages of the irrigated rice culture in Cachoeirinha, RS, Brazil. The spider diversity associated to a rice field was surveyed along different stages of the culture. The studied area belongs to the Estação Experimental do Arroz (EEA), Instituto Rio Grandense do Arroz (IRGA), Cachoeirinha, state of Rio Grande do Sul, Brazil (50 58'21'W; 29 55'30'S). Between October 2004 and June 2005, 17 samples were taken, distributed in three periods: before seeding, during the development of the rice plants and after the harvest. Samples were done in the morning using sweeping nets $(35 \mathrm{~cm}$ diameter), 50 sweeps in each of four randomly chosen transects. A total of 918 spiders were sampled, distributed in 14 families, mostly Araneidae, Anyphaenidae, Oxyopidae and Tetragnathidae. Among the adults, 38 morphospecies were found, the most abundant were Alpaida veniliae (Keyserling, 1865), Tetragnatha nitens (Audouin, 1826), Ashtabula sp.1 and Tetragnatha aff. jaculator, the four together comprising more than $45 \%$ of the adult specimens. Of the species richness estimators used, Chao 1 was closer to the observed richness: $87,4 \%$ of the potentially present species were effectively sampled. Both abundance and species richness showed an increasing trend, accompanying rice development (and thus increasing habitat complexity), with a stern decrease after harvesting. A constant colonization of the habitat is thus postulated, also given the high number of young spiders found at all times. There were no significant correlations between climatic factors (temperature and rainfall) and neither abundance nor species richness, except a positive one between rainfall and richness. Among the functional groups, ambushing hunters dominated, followed by orb-web builders. An analysis of similarity (ANOSIM) found significant differences among the fauna of the three evaluated periods. Thus, system disturbance, in the form of rice sowing and harvesting, brutally altering environmental structure, leads to strong spider diversity changes both in terms of species richness and species composition. The results suggest biodiversity studies in agroecosystems can help us understand not only applied but also basic problems.
\end{abstract}

KEYWORDS. Agroecosystem, biodiversity, spiders.

RESUMO. Com a intenção de conhecer a diversidade da araneofauna relacionada à cultura do arroz, foi realizado um inventário deste agroecossistema ainda pouco estudado em sua biodiversidade. Foram realizadas 17 amostragens na Estação Experimental do Arroz (EEA), do Instituto Rio Grandense do Arroz (IRGA), Cachoeirinha, RS (50'58'21'W; 29 $55^{\circ} 30^{\prime \prime}$ S) entre outubro de 2004 e junho de 2005 , em três períodos: antes do arroz ser semeado, durante o desenvolvimento do arroz e após a colheita. As coletas foram efetuadas no período matinal utilizando rede-de-varredura (35 cm de diâmetro), 50 golpes em cada um dos quatro transectos por amostragem. Coletou-se um total de 918 aranhas distribuídas em 14 famílias, com predomínio de Araneidae, Anyphaenidae, Oxyopidae e Tetragnathidae. Entre os indivíduos adultos, foram determinadas 38 morfoespécies, as mais abundantes Alpaida veniliae (Keyserling, 1865), Tetragnatha nitens (Audouin, 1826), Ashtabula sp.1 e Tetragnatha aff. jaculator, as quatro juntas com mais de $45 \%$ dos espécimes adultos coletados. Dos estimadores de riqueza de espécies utilizados, o que mais se aproximou da riqueza observada foi Chao 1; segundo este, 87,4\% das espécies potencialmente presentes foram amostradas. Os resultados demonstraram que tanto abundância como riqueza tiveram a tendência ao crescimento, acompanhando o desenvolvimento da lavoura de arroz. Uma constante colonização no hábitat foi constatada dado o alto número de aranhas jovens encontradas em todos os períodos. Não foram encontradas diferenças significativas para a correlação entre dados abióticos (temperatura e pluviosidade) com a abundância e a riqueza, exceto pluviosidade vs. riqueza. Entre os grupos funcionais, houve o predomínio das caçadoras emboscadoras, seguido das construtoras de teias orbiculares. A análise de similaridade (ANOSIM) encontrou diferenças significativas entre a fauna dos três períodos avaliados. Assim, a perturbação na forma como o arroz é semeado e colhido altera a estrutura ambiental brutalmente, conduzindo a uma mudança na diversidade de aranhas em termos de riqueza e composição de espécies. Os resultados sugerem a importância de estudos da biodiversidade nos agroecossistemas.

PALAVRAS-CHAVE. Agroecossistema, biodiversidade, aranhas.

Uma das principais causas da perda de biodiversidade é a destruição de habitats resultante da expansão das populações humanas e de suas atividades agrícolas. Mesmo com poucos estudos sobre a fauna relacionada aos agroecossistemas, esses ambientes têm sido cada vez mais vistos sob uma ótica ambiental (ALTIERI $\&$ Nicholls, 2003), servindo a pesquisas que podem não só responder questões ecológicas aplicadas como também básicas.

Segundo Lewinsohn \& Prado (2002), dos inventários de invertebrados no Brasil, apenas 6\% corresponde a Arachnida, destacando-se as aranhas, nas quais se nota uma tendência recente ao aumento. A ordem Araneae é a sétima em diversidade global (CoDDINGTON \& LEVI, 1991), apresentando atualmente 40.462 espécies, incluídas em 3.694 gêneros de 109 famílias (Platnick, 2008), estando entre os mais abundantes invertebrados predadores em ecossistemas terrestres conhecidos (WISE, 1993).

A densidade e diversidade da comunidade de aranhas têm sido intimamente vinculadas à complexidade estrutural do ambiente (RYPSTRA et al., 1999). Apesar de 
sua ubiqüidade e altas densidades (Turnbull, 1973; NyfFelER \& BENZ, 1987), têm sido pouco estudadas em alguns ambientes como os agroecossistemas, especialmente o arroz. Conforme LEWINSOHN \& PRADO (2002), os inventários de animais no Brasil ocorrem em maior quantidade na Floresta Amazônica e em agroecossistemas, entretanto, neste último, são mais pesquisados insetos e moluscos. Nos ecossistemas alterados por atividade humana faltam inventários de táxons que compõem a biota original da região; tais ecossistemas poderiam ser melhor analisados quanto à perda de biodiversidade ou a alterações mais específicas.

GREENSTONE \& SundERLAND (1999) comentaram a necessidade urgente de se utilizar aranhas como agentes de controle biológico no manejo de insetos-praga em agroecossistemas. Além dos estudos com controle biológico, as pesquisas relacionadas à diversidade de aranhas em agroecossistemas também são importantes para observar se o ambiente se manteve ou continua em permanente mudança.

As áreas próximas aos agroecossistemas mantém parte da fauna original e as aranhas apresentam a capacidade para dispersar deste para outros ambientes; alguns estudos demonstram que as aranhas apresentam maior diversidade nas áreas adjacentes do que nas culturas (OrAZE et al., 1988; MuRATA, 1995). Possivelmente as áreas de entorno da lavoura servem como fonte de colonização para o agroecossistema.

Apenas um estudo foi publicado sobre a fauna de aranhas relacionada à cultura do arroz no Rio Grande do Sul: CoRseuIL et al. (1994b) inventariaram - com rede-devarredura e de imersão - aranhas associadas a uma lavoura de arroz irrigado em Itaqui. Na América do Sul, BASTIDAs et al. (1993, 1994) e Medina (1994) pesquisaram aranhas como reguladoras de insetos-praga em lavouras na Colômbia, analisando aspectos relacionados ao controle natural de insetos. Outros estudos que foram realizados na América do Norte envolvem guildas e elaboração de listas de espécies (WoODS \& HARREL, 1976; Young \& EDWARDS, 1990). A Ásia, continente com a maior produção mundial de arroz (IRGA, 2004), registra a maioria das pesquisas com aranhas relacionadas à cultura, tanto para composição de espécies quanto para estrutura das guildas (BARRION, 1999; BAMBARADENIYA \& EDIRISINGHE, 2001; Bambaradeniya et al., 2004; Patel et al., 2004; VIJAYKUMAR, 2004).

Dos trabalhos relacionados à diversidade de aranhas em agroecossistemas, vários sobre a cultura do arroz, há somente um para o Brasil, no Rio Grande do Sul. Os dados conhecidos são pouco significativos para uma região onde a orizocultura predomina em grandes extensões no Estado. O objetivo deste trabalho foi analisar a diversidade da assembléia de aranhas associada à lavoura de arroz, seus grupos funcionais e as alterações na fauna araneológica ao sofrer a influência dos distúrbios causados pelo plantio e colheita do arroz.

\section{MATERIAL E MÉTODOS}

As amostragens foram efetuadas na Estação Experimental do Arroz (EEA) (5058'21',W; 2955'30'S), do Instituto Rio Grandense do Arroz (IRGA), localizada no município de Cachoeirinha, RS. A EEA está situada às margens do rio Gravataí, com uma área de 172 hectares (IRGA, 2004). O município de Cachoeirinha pertence à Depressão Central, região fisiográfica que ocupa a porção mediana do Estado do Rio Grande do Sul (Teixeira et al., 1986). Conforme IRGA (2004), a Depressão Central é responsável por 14,53\% da produção total do arroz do RS.

As coletas foram realizadas de 20/10/2004 a 06/06/2005, com intervalo médio entre cada período amostral de 11,3 dias, visando cobrir as diferentes fases do desenvolvimento do arroz. Foram efetuadas 17 amostragens: as três primeiras antes do arroz ter sido semeado ("antes"), da $4^{\mathrm{a}}$ até a $14^{\mathrm{a}}$ no período de crescimento das plantas ("durante") e as três últimas coletas após o arroz ter sido colhido, na chamada reesteva ("depois").

Utilizou-se quatro transectos, sorteados ao acaso em cada coleta, tomando o cuidado para não utilizar os mesmos usados na coleta anterior. Foram amostradas duas áreas contíguas, separadas por um pequeno canal de aproximadamente dois metros de largura, sendo dois transectos em cada área. O sistema de plantio do arroz foi o convencional e o cultivar, BR IRGA 410. No período de preparação da área onde foi semeado o arroz, entre a $3^{\mathrm{a}} \mathrm{e}$ a $4^{\mathrm{a}}$ coleta, foram aplicados os herbicidas FACET 750 PM (300 g por hectare), STAM 480 (4 litros por hectare) e Sirius $250 \mathrm{SC}(60 \mathrm{ml}$ por hectare). Durante o desenvolvimento da lavoura não foi utilizado nenhum produto fitossanitário, com o intuito de não influenciar a comunidade de artrópodes na área.

As coletas foram realizadas com rede-de-varredura (35 cm de diâmetro). Em cada transecto efetuou-se 50 golpes com a rede em movimentos de avanço pendulares. O material coletado foi acondicionado em sacos plásticos de 30 litros, lacrados, etiquetados com os dados de coleta e guardados em local sem contato direto com o sol e altas temperaturas. As coletas foram realizadas pela manhã, entre 8 e 11 horas, com a finalidade de minimizar os efeitos da migração de aranhas para o estrato inferior da vegetação pelas altas temperaturas do período do meio dia (Dumas et al., 1964). Para a conservação do material, foi utilizado álcool etílico a $80 \%$.

A identificação e catalogação foi realizada no Museu de Ciências Naturais da Fundação Zoobotânica do Rio Grande do Sul. Alguns indivíduos da família Thomisidae foram tombados na coleção do Museu de Ciências e Tecnologia da Pontifícia Universidade Católica do RS. Todas as aranhas capturadas foram identificadas em nível de família e somente os indivíduos adultos separados em morfoespécies.

Para análise dos dados utilizou-se curvas do coletor e estimadores analíticos de riqueza de espécies (Chao 1, Jacknife 1, Bootstrap e Michaelis-Menten) para verificar a suficiência amostral. Para isto foi usado o programa EstimateS versão 7.5 (CoLWELl, 2005) usando 500 aleatorizações.

Para averiguar diferenças estatísticas por amostragem entre os períodos (antes da semeadura, durante o desenvolvimento da lavoura e depois da colheita), para as variáveis riqueza de espécies, abundância e equitabilidade, foi realizada uma MANOVA 
(análise de variância multivariada) com a utilização do software SPSS ${ }^{\circledR}$. Foram realizadas análises de correlação paramétrica entre a riqueza e abundância e dados abióticos (temperatura média e pluviosidade) sobre a região. Estes valores foram disponibilizados pela FEPAGRO (Fundação Estadual de Pesquisa Agropecuária).

As aranhas foram classificadas a partir de suas estratégias de caça em grupos funcionais (guildas), com o intuito de conhecer quais ocorrem na lavoura, seguindo UETz et al. (1999) e HöFER \& BRESCOVIT (2001). Cálculos de diversidade foram realizados a partir do programa PASt (Paleontological Statistics, versão 1.34) (HAMMER \& HARPER, 2005), utilizando os índices de Shannon-Wiener (H') e "Evenness".

Para comparar a composição da araneofauna, foram realizadas análises de agrupamento (UPGMA) utilizando os índices de Jaccard e Morisita, com o objetivo de procurar padrões temporais entre as composições das espécies. Para verificar a existência de diferenças estatísticas entre essas composições, para ambos os índices, foram aplicadas duas ANOSIM de um fator, com correção de Bonferroni para repetidos testes. Para estes cálculos foi utilizado o programa PASt v.1.34.

Para verificar diferenças significativas entre o tamanho das aranhas adultas das diferentes espécies entre os períodos da lavoura, foi aplicada uma ANOVA de um fator e teste de Tukey para comparação múltipla. O comprimento corporal foi medido excluindo as quelíceras e as fiandeiras.

\section{RESULTADOS E DISCUSSÃO}

Fauna de aranhas da cultura do arroz. Foram capturadas 918 aranhas, incluindo jovens e adultas, distribuídas em 14 famílias. O predomínio foi de aranhas jovens (713 indivíduos) sobre adultas (205), destas 122 fêmeas e 83 machos. Entre as adultas, foram determinadas 38 morfoespécies.

Das famílias de aranhas, houve predomínio de Araneidae ( $\mathrm{N}=177$ ), Anyphaenidae (126), Oxyopidae (124) e Tetragnathidae (103), somando as quatro mais de $57 \%$ do total de exemplares coletados. Corinnidae, Philodromidae, Theridiidae, Gnaphosidae e Pisauridae representaram menos de $1 \%$ do total amostrado (Tab. I). Com exceção de Theridiidae, todas foram representadas exclusivamente por indivíduos jovens (Fig. 1).

Araneidae foi a única família registrada nas 17 amostragens efetuadas (Tab. I); o maior número de morfoespécies ocorreu em Linyphiidae (12) e Araneidae (6), a primeira sendo apenas a sétima em abundância. Miturgidae, Oxyopidae e Theridiidae apresentaram somente uma morfoespécie cada (Tab. II). Linyphiidae, conforme THORBEK et al. (2004), consegue viver em áreas perturbadas por atividade agrícola. Em estudos realizados em agroecossistemas na América do Sul, os linifídeos têm sido representados por poucas morfoespécies se comparadas com os demais grupos (Aguilar, 1989; Corseuil et al., 1994a); entretanto, na Europa a representatividade de morfoespécies nestes ambientes é bem maior (Pommeresche, 2004; PekÁr \& KocoukeK, 2004).
Em lavouras de arroz na América do Sul, com a mesma metodologia de coleta, a quantidade de famílias foi similar. CoRsEuIL et al. (1994b) encontrou nove e BAstidas et al. $(1993,1994)$ obtiveram 11. Em trabalhos conduzidos em outras regiões, o número também foi baixo, muitas vezes menos de 10 (WoODS \& HARREL, 1976; OrAZE et al., 1988; Murata, 1995; ViJAYKUMAR, 2004).

Das 38 morfoespécies apontadas neste estudo, as mais abundantes foram Alpaida veniliae (Keyserling, 1865) $(\mathrm{N}=50)$, Tetragnatha nitens (Audouin, 1826) (17), Ashtabula sp.1 (15) e Tetragnatha aff. jaculator (13), somando 46,35\% da abundância total. A grande maioria (20 morfoespécies) não alcançou $1 \%$ do total (Tab. II). O número de morfoespécies registradas neste trabalho (38) foi próximo, ou maior, que outros estudos em áreas de plantações de arroz (OrAZE et al., 1988 (10 morfoespécies), Bastidas et al., 1993 (27), CoRseuil et al., 1994b (20), Medina, 1994 (21), Patel et al., 2004 (37); porém, em pesquisas no continente asiático, a quantidade é surpreendentemente maior (LEE \& KIM, 2001 (175); BAMBARADENIYA et al., 2004 (59), talvez pela amostragem ser mais abrangente em termos de espaço e tempo.

A maior abundância e freqüência (11 coletas) de Alpaida veniliae na lavoura de arroz não foi inesperada, visto que esta espécie já havia sido registrada para este agroecossistema por CoRseuil et al. (1994b) e para Medina (1994), na Colômbia, foi a mais abundante nesta cultura. Segundo LEvi (1988), A. veniliae é encontrada próxima à água, onde constrói teias a um metro do solo. Efetivamente, esta espécie foi observada sempre em teias entre as plantas de arroz, em lugares permanentemente com água no solo. Também é registrada na soja no Brasil e Argentina (CORSEUIL et al., 1994a; LILJESTHRÖM et al., 2002).

Alguns gêneros e espécies registrados por Corseuil et al. (1994b) para o arroz, também foram coletados na presente pesquisa. Entre as três espécies identificadas nominalmente (Alpaida veniliae, Sanogasta (Gayenna) maculatipes (Keyserling, 1878) e Oxyopes salticus Hentz, 1845) por CoRsEUIL et al. (1994b), todas ocorrem também na lavoura de arroz aqui estudada.

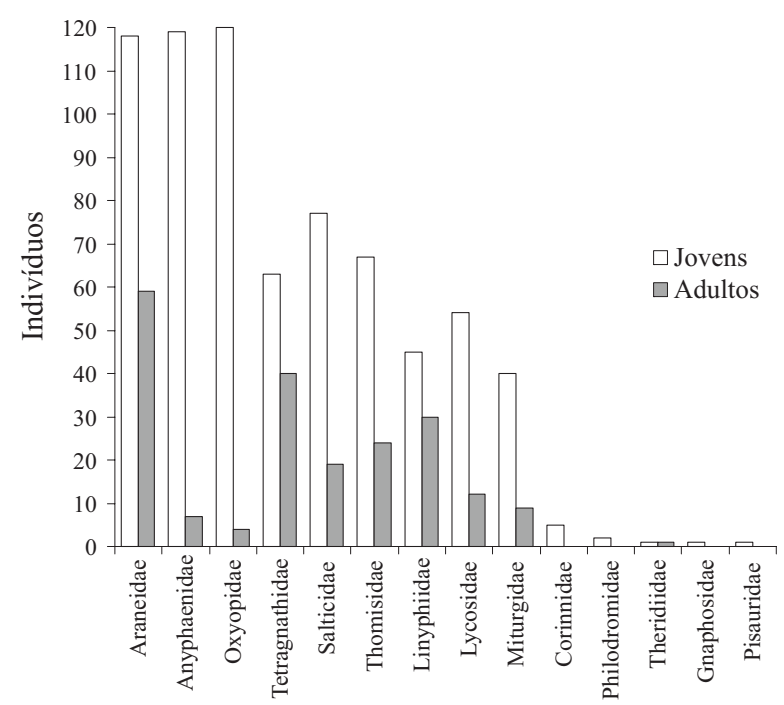

Fig. 1. Abundância de aranhas jovens e adultas, por família, encontradas entre 20/10/2004 a 6/06/2005 em um cultivo de arroz na Estação Experimental do Arroz, IRGA, Cachoeirinha, RS, Brasil. 
Possivelmente são espécies que ocorram também em outras lavouras de arroz no Rio Grande do Sul e até mesmo no restante do Brasil.

Suficiência amostral e estimadores analíticos de riqueza de espécies. A curva de acumulação de espécies não alcançou uma assíntota, tendendo a continuar em ascensão (Fig. 2). Até a $16^{\mathrm{a}}$ coleta ainda foram incluídas espécies diferentes na lavoura de arroz, indicando que a amostragem foi incompleta e/ou que há um constante fluxo de colonização de espécies na área. Assim como a curva do coletor, as curvas dos estimadores de riqueza de espécies também seguiram o mesmo padrão, não alcançando assíntotas claras (Fig. 2). Dos estimadores

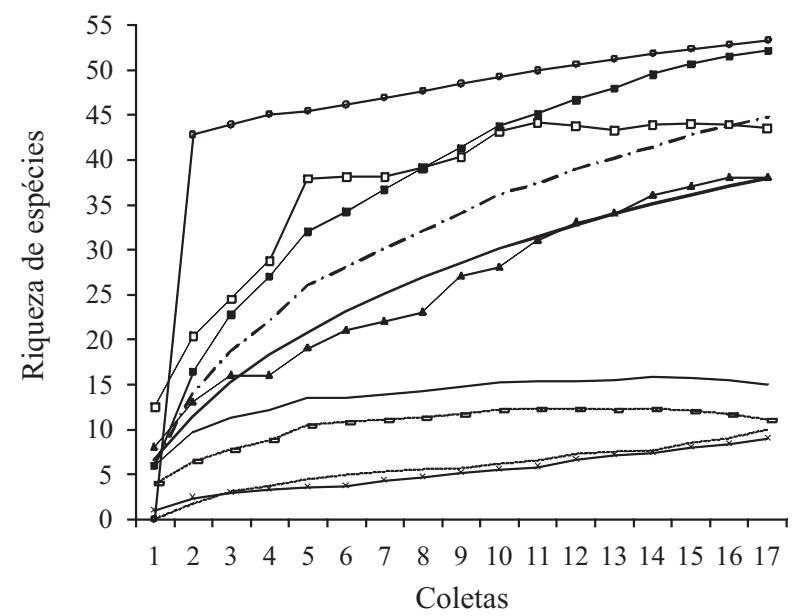

$\begin{array}{lll}\rightarrow \text { S (corrigida) } & \rightarrow \text { S (observada) } & \rightarrow \text { Chao } 1 \\ \rightarrow \text { Jack 1 } & - \text { - Bootstrap } & \rightarrow \text { MM } \\ \rightarrow \text { Singletons } & \sim \text { Doubletons } & - \text { Uniques } \\ - \text { Duplicates } & & \end{array}$

Fig. 2. Curvas de estimativa de riqueza de espécies, da riqueza observada, corrigida (randomizadas 500 vezes), singletons, doubletons, uniques e duplicates das 17 amostragens realizadas no período de 20/10/2004 a 6/06/2005 na Estação Experimental do Arroz, IRGA, Cachoeirinha, RS, Brasil (S, riqueza de espécies; Jack 1, Jacknife de primeira ordem; MM, Michaelis-Menten). utilizados, o que mais se aproximou da riqueza observada e de uma estabilização foi Chao $1(43,5)$; segundo este estimador, $87,4 \%$ das espécies da área teriam sido amostradas; após aparece Bootstrap $(44,68 ; 85,1 \%)$. No outro extremo, o índice de Michaelis-Menten estimou um valor de 53,28 espécies, representando 71,3\% das espécies amostradas e Jacknife 1 estimando 52,12 espécies, representando 72,9\% das espécies encontradas (Fig. 2).

Os estimadores de riqueza são calculados a partir da quantidade de singletons e/ou doubletons, espécies raras (representadas por somente um ou dois indivíduos) ou dos uniques e duplicates, espécies infreqüentes (representadas em somente uma ou duas amostras) (Fig. 2). Do total de morfoespécies, 11 são representadas por singletons, somando 28,9\% do total. Já os doubletons, em menor quantidade, somaram nove morfoespécies $(23,7 \%)$. Os uniques totalizaram 15 morfoespécies e os duplicates 10. CHAO (1984) cita que os estimadores avaliam a fauna disponível para o método; mais espécies podem assim ser esperadas para área em questão. Possivelmente os táxons amostrados neste estudo não demonstram a totalidade de espécies ocorrentes no arroz; este fato é corroborado pelo padrão encontrado a partir da curva do coletor.

Diversidade de aranhas nos períodos da cultura do arroz: dinâmica da assembléia. Para as três variáveis de diversidade analisadas juntas (abundância, riqueza e equitabilidade), os períodos diferiram significativamente (Pillai's Trace $=0,266 ; \mathrm{F}=2,811 ; \mathrm{gl}=6 ; 110 ; p=0,014$ ). A média final para a abundância foi de 13,5 aranhas por transecto $(\mathrm{EP} \pm 1,5)$ entre todas as coletas. Entre os períodos, as variações foram maiores e as diferenças significativas $(\mathrm{F}=6,609 ; \mathrm{gl}=2 ; 56 ; p=0,003)$, com o período "durante" a cultura do arroz com maior número médio de aranhas amostradas. Efetivamente, a quantidade de aranhas aumentou (Fig. 3) durante o desenvolvimento das plantas de arroz, havendo um lento acréscimo na quantidade de indivíduos no início da cultura mas aumentando em relação ao seu final (a partir da $10^{\mathrm{a}}$ coleta).

Tabela I. Número de indivíduos (adultos e jovens) por família e grupos funcionais (guildas) encontrados entre 20/10/2004 a 6/06/2005, nas 17 coletas efetuadas na Estação Experimental do Arroz, IRGA, Cachoeirinha, RS, Brasil (CTO, construtoras teias orbiculares; CTI, construtoras teias irregulares; CCO, caçadoras corredoras; CEM, caçadoras de emboscada).

\begin{tabular}{|c|c|c|c|c|c|c|c|c|c|c|c|c|c|c|c|c|c|c|c|c|}
\hline \multirow[t]{2}{*}{ Famílias } & \multirow[t]{2}{*}{ Guildas } & \multicolumn{17}{|c|}{ Coletas } & \multirow[b]{2}{*}{ Total } & \multirow[b]{2}{*}{$\%$} \\
\hline & & 1 & 2 & 3 & 4 & 5 & 6 & 7 & 8 & 9 & 10 & 11 & 12 & 13 & 14 & 15 & 16 & 17 & & \\
\hline Araneidae & $\mathrm{CTO}$ & 4 & 5 & 8 & 1 & 1 & 7 & 18 & 14 & 15 & 17 & 21 & 19 & 21 & 13 & 11 & 1 & 1 & 177 & 19,28 \\
\hline Anyphaenidae & $\mathrm{CCO}$ & & 1 & 1 & & & 1 & & & 6 & 4 & 14 & 19 & 39 & 33 & 6 & 1 & 1 & 126 & 13,72 \\
\hline Oxyopidae & CEM & 2 & 1 & 5 & & 1 & & & 2 & 5 & 3 & 15 & 13 & 36 & 34 & 7 & & & 124 & 13,51 \\
\hline Tetragnathidae & СТO & 6 & & 2 & & 13 & 22 & 6 & 14 & 16 & 19 & & 1 & 2 & & 2 & & & 103 & 11,22 \\
\hline Salticidae & CEM & 1 & 1 & 3 & & & 2 & & 1 & 6 & 7 & 17 & 15 & 20 & 12 & 6 & 4 & 1 & 96 & 10,46 \\
\hline Thomisidae & CEM & 1 & 8 & 9 & 2 & 1 & 1 & & 6 & 2 & 5 & 8 & 11 & 9 & 19 & 5 & 3 & 1 & 91 & 9,91 \\
\hline Linyphiidae & CTI & 14 & 3 & 9 & & & & 1 & 2 & & 3 & 18 & 14 & 3 & 3 & 4 & & 1 & 75 & 8,17 \\
\hline Lycosidae & $\mathrm{CCO}$ & 2 & & & 1 & 1 & & 2 & & 2 & 18 & 6 & 15 & 3 & 10 & 1 & 1 & 4 & 66 & 7,19 \\
\hline Miturgidae & $\mathrm{CCO}$ & & 1 & & & & & & & & 7 & 6 & 12 & 11 & 11 & 1 & & & 49 & 5,34 \\
\hline Corinnidae & $\mathrm{CCO}$ & & & & & & & & & & & 1 & & & 4 & & & & 5 & 0,54 \\
\hline Philodromidae & CEM & & & & & & & & & & & & 2 & & & & & & 2 & 0,22 \\
\hline Theridiidae & CTI & & 1 & & & & & & & & & & & & & & 1 & & 2 & 0,22 \\
\hline Gnaphosidae & $\mathrm{CCO}$ & & & & & & & & & & & & & & & 1 & & & 1 & 0,11 \\
\hline Pisauridae & CEM & & & & & & & & & & & & 1 & & & & & & 1 & 0,11 \\
\hline Total & & 30 & 21 & 37 & 4 & 17 & 33 & 27 & 39 & 52 & 83 & 106 & 122 & 144 & 139 & 44 & 11 & 9 & 918 & 100 \\
\hline
\end{tabular}


Tabela II. Lista das morfoespécies, separadas em número de indivíduos (somente aranhas adultas), nas 17 coletas realizadas entre 20/10/ 2004 a 6/06/2005, na Estação Experimental do Arroz, IRGA, Cachoeirinha, RS, Brasil.

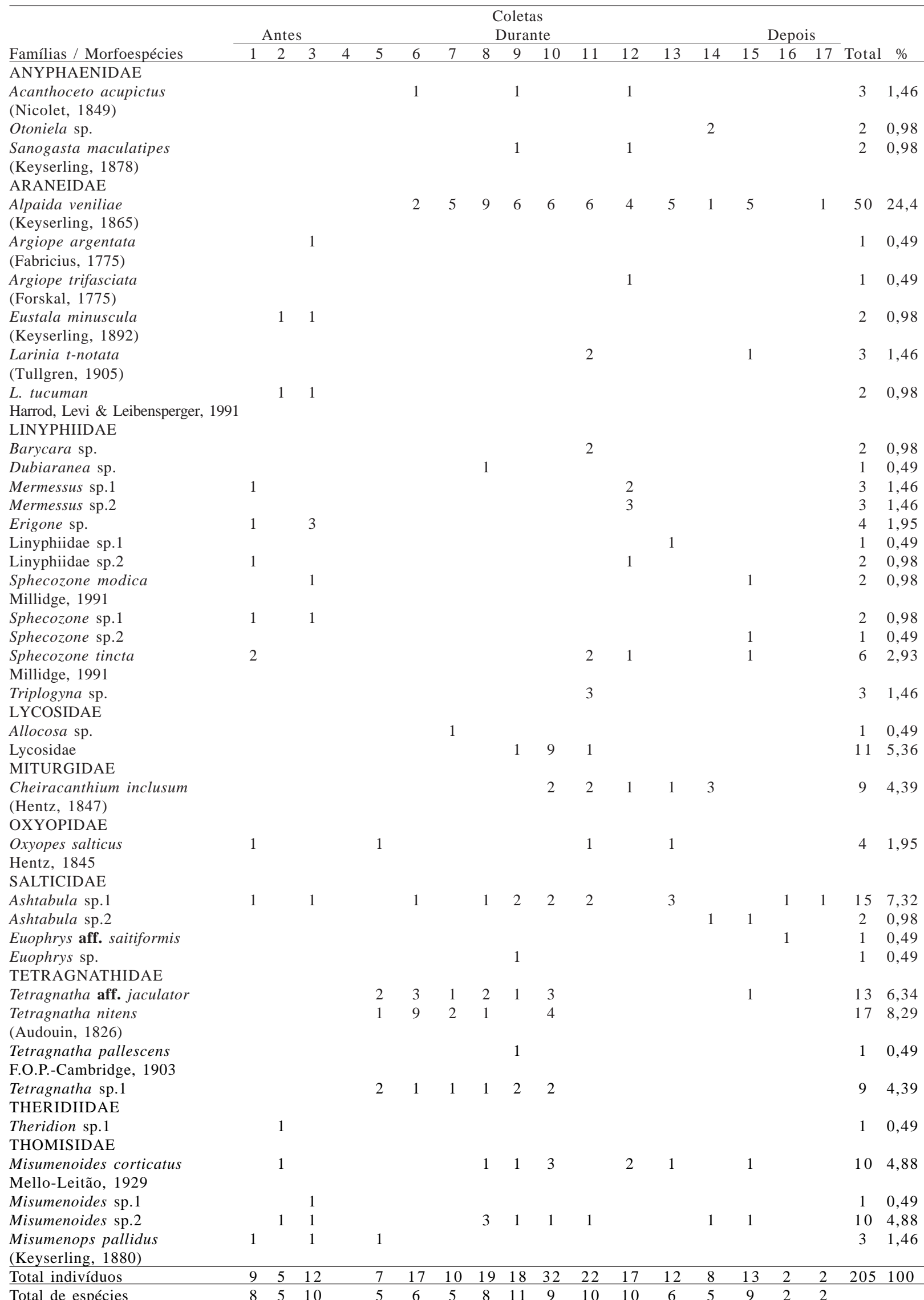


Após o preparo da área da lavoura (entre a $3^{\mathrm{a}}$ e $4^{\mathrm{a}}$ coleta), o ambiente se torna praticamente inviável para a sobrevivência das aranhas, porém, a seguir, a abundância ascende, no decorrer do desenvolvimento das plantas, que passam a servir de substrato. Ambalagan \& NARAYANASAMY (1999) registraram a flutuação populacional das aranhas no arroz na Índia, indicando uma relação entre o aumento da abundância e o crescimento do arroz na lavoura; mesmo padrão encontrado no presente estudo.

Corseuil et al. (1994b) observaram que o número de aranhas encontradas em cada coleta variou muito: até o meio do período de amostragem foram coletadas as maiores abundâncias e, no final das coletas, a quantidade decresceu. Percebe-se aqui que nas últimas três coletas (Fig. 3), após o arroz ter sido colhido, novamente a abundância diminuiu; nesta etapa da lavoura mais uma vez o substrato é retirado e o próprio processo de colheita (passagem de máquinas, tratores, caminhões) deve influenciar na redução das aranhas agindo como um distúrbio intenso.

A coleta mais rica em espécies foi a $9^{\mathrm{a}}$, com 11 espécies registradas; a mais pobre foi a $4^{\mathrm{a}}$ coleta, sem nenhuma espécie (Fig. 3). No entanto, os valores de riqueza antes da semeadura foram também elevados, com um pico na riqueza $(S=10)$, na $3^{\text {a }}$ coleta. Graças a este padrão, o período "durante" não foi significativamente mais rico que os períodos "antes" e "depois" (MANOVA, $\mathrm{F}=2,969 ; \mathrm{gl}=2 ; 56 ; p=0,060)$. As aranhas formavam uma assembléia rica antes do distúrbio da aragem e semeadura, sofrendo uma queda quando com essas alterações, gradativamente recuperando sua riqueza com o crescimento das plantas de arroz até a colheita, quando novamente este distúrbio diminui o número de espécies presentes.

Muitas aranhas vivem restritas a determinados ambientes e, em muitos casos, suas limitações podem estar relacionadas a fatores biológicos como tipo de vegetação, quantidade de alimento, competidores e inimigos (FoELIX, 1982); em muitos casos, a abundância de cada espécie de aranha está relacionada com a complexidade do habitat (RYPSTRA et al. 1999; GunNARsson, 1990). Um processo de gradativo aumento da complexidade do habitat pode estar ocorrendo ao longo do desenvolvimento das plantas de arroz ("durante"), explicando a variação observada na riqueza de espécies.

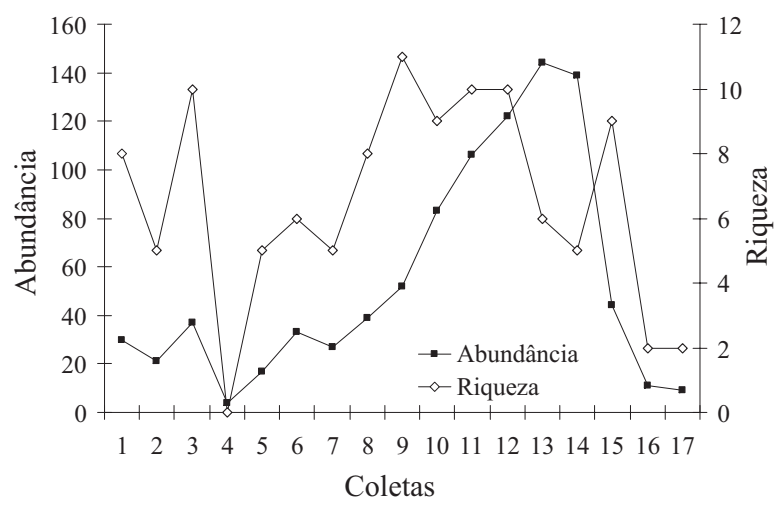

Fig. 3. Abundância de aranhas (adultas e jovens) e riqueza (somente adultas) nas 17 coletas efetuadas entre 20/10/2004 a 6/06/2005 na Estação Experimental do Arroz, IRGA, Cachoeirinha, RS, Brasil.
Um fator que influenciou nos picos de abundância registrados foi o número de aranhas jovens $(78,7 \%$ do total de espécimes), que tiveram maiores valores na $14^{\mathrm{a}} \mathrm{e}$ $15^{\mathrm{a}}$ coletas. A quantidade de adultos não foi tão elevada, variando pouco no decorrer das amostragens. Tanto no arroz como em outros agroecossistemas, a maioria das aranhas obtidas são de indivíduos jovens (CORSEUIL et al., 1994a, b; NuNEZ \& BALLINAS, 1998).

É possível supor que nas áreas de arroz haja uma grande colonização de aranhas a partir de dispersão aérea (balonismo) que, conforme vAN WingERdEN \& VugTs (1974) e HoRner (1975), em muitas espécies é utilizada pelos jovens. De acordo com BAMBARADENIYA \& EDIRISINGHE (2001), quando uma nova lavoura é estabelecida, tanto aranhas adultas quanto jovens colonizam os campos de arroz por dispersão aérea, a partir de áreas circunvizinhas. No arroz, devido à inundação imposta às áreas cultivadas, a dispersão via terrestre pode ser dificultada, explicando a prevalência de jovens.

Os dados relativos aos jovens, muitas vezes desconsiderados em trabalhos pela dificuldade de identificação, aqui elucidam o processo de montagem da assembléia observado no incremento do número de indivíduos e espécies ao longo do crescimento das plantas de arroz. Este acúmulo de indivíduos de diferentes espécies sugere também que o processo de entrada na assembléia pode ser constante. Isto deve estar aliado a um mecanismo de permanência dos dispersores no ambiente, talvez conforme a oferta de alimentos. Por exemplo, BAMBARADENIYA \& EDIRISINGHE (2001) demonstraram que, em lavouras de arroz, a abundância das aranhas aumentou conforme o número de insetos também aumentava.

Fatores abióticos influenciando a fauna de aranhas. A comparação entre os dados abióticos (temperatura e pluviosidade) com a abundância e a riqueza demonstrou que as correlações não foram significativas, com exceção da pluviosidade com a riqueza. A temperatura não influenciou nem na abundância $\left(\mathrm{r}^{2}=0,031 ; p=0,83\right)$ nem na riqueza $\left(\mathrm{r}^{2}=0,076 ; p=0,28\right)$, padrão semelhante ao encontrado por VIJAYKUMAR (2004) na Índia. A precipitação também não mostrou valores significativos correlacionados com a variável abundância $\left(\mathrm{r}^{2}=0,034 ; p\right.$ $=0,47)$; no entanto, a diferença foi estatisticamente significativa para a riqueza $\left(\mathrm{r}^{2}=0,038 ; p=0,02\right)$. A pluviosidade teve um padrão inconstante, visto que no período em que foram realizadas as amostragens, o Rio Grande do Sul passou por intensa estiagem e a quantidade de chuva variou muito, o que pode ter influenciado na correlação: os únicos valores elevados de chuva ocorreram na $9^{\mathrm{a}}$ e $15^{\mathrm{a}}$ coleta e nas duas ocorreram picos de riqueza.

Guildas de aranhas na cultura do arroz. A classificação das aranhas em grupos funcionais (guildas) (Tab. I) demonstrou o predomínio das caçadoras emboscadoras $(34,2 \%)$. Após as construtoras de teias orbiculares $(30,5 \%)$, as caçadoras corredoras $(26,9 \%)$ e, por último, as construtoras de teias irregulares $(8,4 \%)$. A prevalência das caçadoras emboscadoras teve grande influência da alta abundância de Oxyopidae, mesmo representada quase que exclusivamente por aranhas imaturas e pelas famílias Thomisidae e Salticidae, bastante abundantes. 
As construtoras de teias orbiculares foram representadas por duas famílias, Araneidae e Tetragnathidae, a primeira, a mais abundante de todas; são famílias comuns em áreas de lavouras, presumivelmente pelas plantas do arroz serem bons locais para a construção das teias e também pelo elevado número de presas que ocorrem nestas plantações. BAMBARADENIYA \& EDIRISINGHE (2001) observaram que as construtoras de teias orbiculares predam principalmente insetos das ordens Orthoptera, Lepidoptera e Homoptera, sendo a maioria pragas em lavouras no Sri Lanka. As caçadoras corredoras agrupam um alto número de famílias (5), o que é inesperado, pois o solo da cultura do arroz é inundado a maior parte do tempo. Construtoras de teias irregulares foi o grupo menos freqüente, com somente Theridiidae e Linyphiidae. BAmbaradenIYA \& EDIRISINGHe (2001) verificaram, como guilda mais abundante no arroz, as construtoras de teias irregulares, relacionado principalmente à grande quantidade de aranhas das famílias supracitadas. Na Ásia, Theridiidae parece comum nas lavouras de arroz, fato não constatado no presente estudo.

LEE \& KIM (2001) separaram as aranhas em duas guidas - construtoras de teias e caçadoras - considerando como predadoras mais efetivas o segundo grupo. BARRION (1999), nas Filipinas, também separou nestes dois grupos, com predomínio das construtoras de teias na lavoura de arroz e áreas adjacentes, no entanto, como método, utilizou máquina de sucção (D-vac). Nos Estados Unidos, conforme YouNG \& EDWARDS (1990), nos agroecossistemas em geral, a guilda mais abundante foi a das errantes, que não constroem teias, o contrário das guildas encontradas em outros ecossistemas; segundo o mesmo estudo, esta diferença pode ser atribuída pela característica das guildas associadas com a dispersão e habilidade de sobrevivência em locais que sofrem distúrbios.

UETZ et al. (1999) analisaram os grupos funcionais abordados por Young \& EDWARDS (1990) e observaram que as construtoras de teias orbiculares são as mais abundantes, seguidas das emboscadoras, padrão diferente do encontrado nesta pesquisa, possivelmente devido às variações da fauna da Região Neártica para a Neotropical.

Distribuição de abundância entre espécies de aranhas e índices de diversidade. Ao avaliarmos a curva de distribuição de abundância geral, uma espécie foi marcadamente dominante, algumas espécies intermediárias e várias raras no final da curva (Fig. 4). O valor da equitabilidade entre os períodos (Evenness: antes $=0,903$; durante $=0,519$; depois $=0,756$ ), foi baixo em "durante" devido à dominância de $A$. veniliae, que influenciou também no baixo valor de Shannon-Wiener $\left(H^{\prime}=2,677\right)$, comparando com "antes" $\left(H^{\prime}=2,672\right)$ a partir da riqueza de espécies destes períodos. Ambos ficaram distantes do período "depois" $\left(\mathrm{H}^{\prime}=2,119\right)$ pelo baixo número de espécies apresentado.

Composição da assembléia de aranhas na cultura do arroz. Analisando a heterogeneidade temporal em termos da composição de espécies da lavoura estudada, utilizando como fator os períodos, nota-se que a partir do índice de similaridade de Jaccard (Fig. 5), as áreas "durante" formaram um agrupamento isolado, com os maiores valores de similaridade, os períodos "depois" e "antes" também formaram clusters isolados. Utilizando o índice de Morisita (Fig. 6), percebe-se a formação de um agrupamento entre os períodos "durante" e "depois" e um outro cluster do período "antes". No agrupamento do período "durante" e "depois", a maior similaridade foi encontrada para três períodos "durante", reunidos pelos altos números de indivíduos de mesmas espécies que o índice de Morisita leva em consideração (MAGURRAN, 1988). Assim como este grande grupo, o agrupamento formado do período "antes" foi influenciado pela presença das espécies dominantes, no primeiro caso A. veniliae e no segundo Erigone sp. 1, pois o índice de Morisita é sensível à abundância dessas espécies mais dominantes (MagurRan, 1988). Um cluster foi formado entre A4Depois e A2Antes, mesmo em períodos distintos e com faunas heterogêneas, pois a ausência em ambos da espécie dominante de cada período influenciou na formação do agrupamento.

A partir do teste de ANOSIM, foi constatado que os resultados acima descritos foram estatisticamente diferentes para ambos índices de similaridade, Jaccard ( $\mathrm{R}$ $=0,441, p=0,0016)$ e Morisita $(\mathrm{R}=0,3519, p=0,011)$ (mesmo aplicando a correção de Bonferroni, $\alpha=0,025$ ), demonstrando que a araneofauna entre os períodos foi distinta e a pouca similaridade, possivelmente, foi influenciada pelas diferenças fisionômicas entre os períodos da área da cultura, refletidos na fauna de aranhas ali existente.

Tal conclusão pode dar peso à idéia exposta acima de que muitas espécies de aranhas são ambienteespecíficas não só para tipo de vegetação como para o grau de complexidade do habitat; aliado a isto, diferenças na capacidade de colonização destes ambientes podem estar afetando quais espécies ocorrem em cada momento na área. Estudos futuros podem buscar distinguir a importância destes dois fatores, mas para tal são necessários também dados biológicos mais completos sobre as espécies que ocorrem na área estudada.

Tamanho corporal das aranhas na cultura do arroz. O tamanho médio dos indivíduos adultos coletados na área da lavoura de arroz foi de $5,62 \mathrm{~mm}( \pm 0,17 ; \mathrm{N}=205)$; no entanto, foram coletadas aranhas de 0,5 até $11 \mathrm{~mm}$. Nos três períodos os tamanhos médios variaram nas espécies encontradas (antes: $2,81, \mathrm{EP} \pm 0,28$; durante: 4,61 , $\pm 0,52$; depois: $3,79, \pm 0,53$; Fig. 7 ). A diferença foi

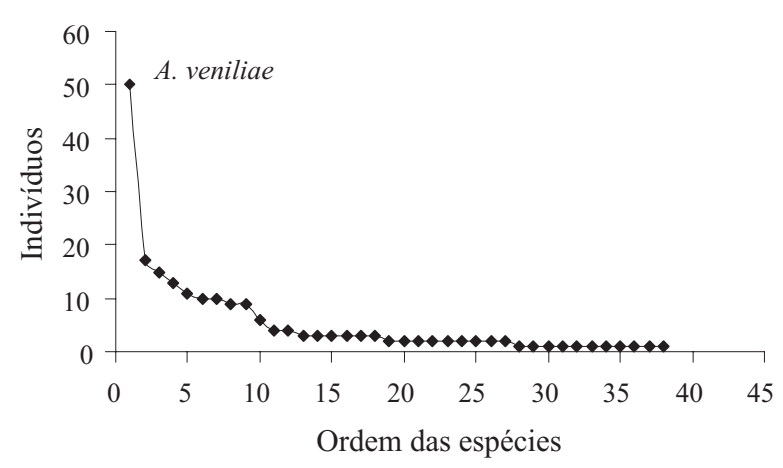

Fig. 4. Curva de distribuição de abundância das morfoespécies de aranhas encontradas nas 17 amostragens efetuadas no período de 20/10/2004 a 6/06/2005 na Estação Experimental do Arroz, IRGA, Cachoeirinha, RS, Brasil. 

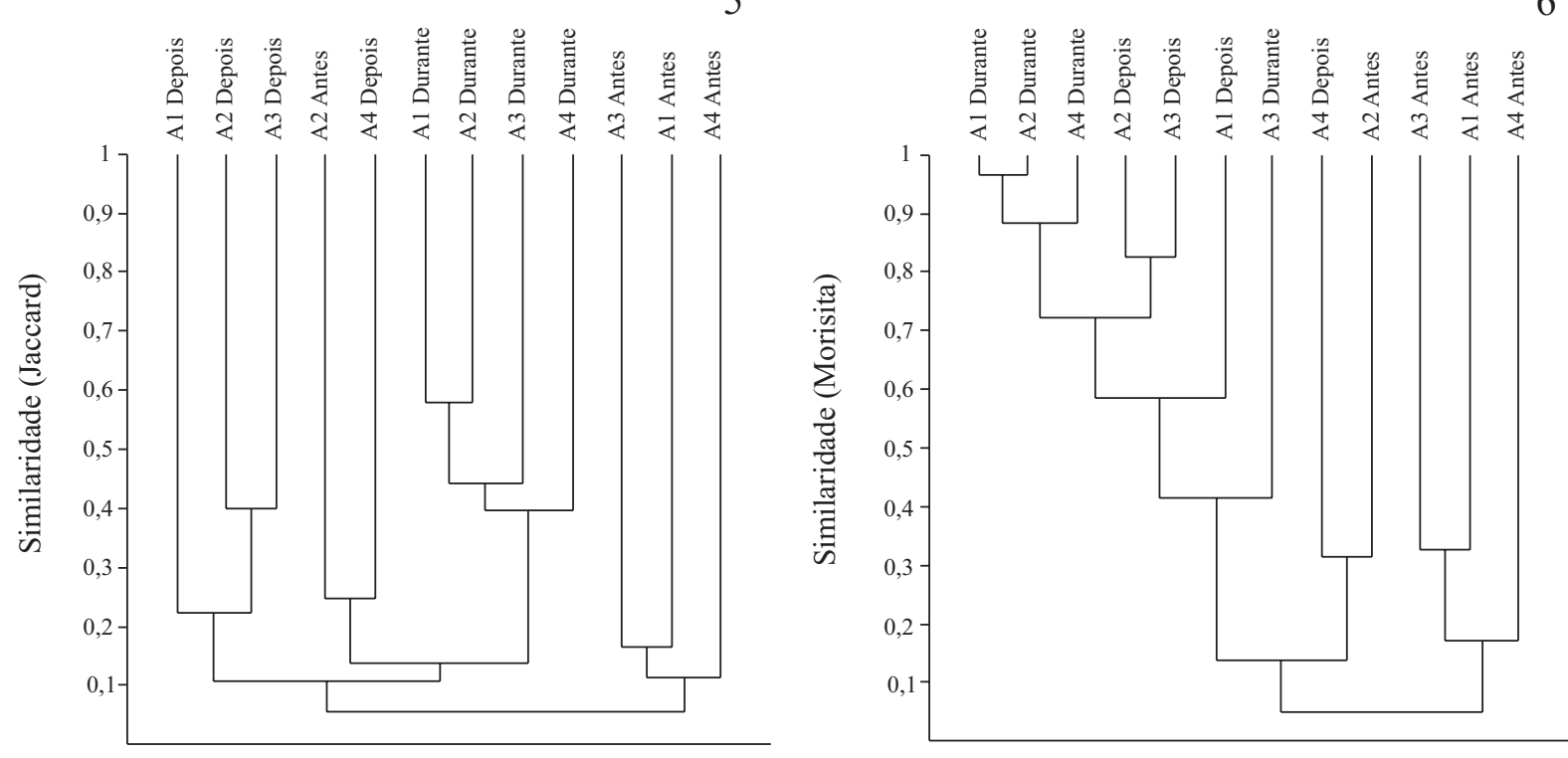

Figs. 5, 6. Análise de agrupamento (UPGMA) por similaridade utilizando as morfoespécies amostradas nos diferentes períodos estudados na Estação Experimental do Arroz, IRGA, Cachoeirinha, RS, Brasil: 5, Índice de Jaccard; 6, Índice de Morisita (A1 - A4, transectos).

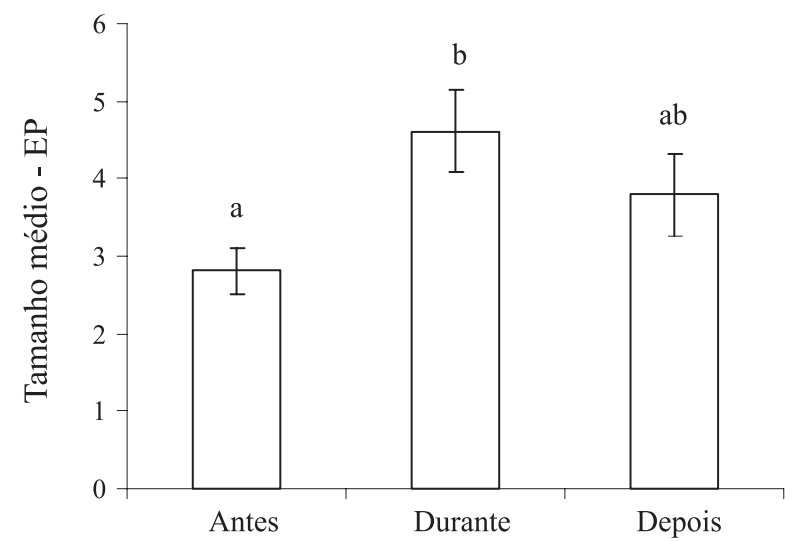

Fig. 7. Tamanhos médios $(\mathrm{mm})$ das aranhas adultas e erro padrão em cada um dos períodos estudados, nas 17 coletas efetuadas entre 20/10/2004 a 6/06/2005 na Estação Experimental do Arroz, IRGA, Cachoeirinha, RS, Brasil (Divergências entre as letras indicam diferença significativa, teste Tukey para $p<0,05)$.

estatisticamente significativa $(\mathrm{F}=4,69 ; \mathrm{gl}=2 ; 52 ; p=0,013)$ e, na aplicação do teste de Tukey para comparação múltipla, foi significativa entre "antes" x "durante" $(p=0,02)$.

As diferenças entre a fauna de cada período influenciaram nas variações encontradas nos tamanhos médios; as maiores espécies foram encontradas "durante", como os maiores indivíduos de Tetragnatha nitens (8 - $11 \mathrm{~mm}$, que só foram registrados neste período, possivelmente relacionada com suas teias orbiculares, que necessitam de um substrato para a sua construção), Alpaida veniliae (8,5 - $11 \mathrm{~mm})$, Cheiracanthium inclusum (Hentz, 1847) (7 - 7,5 mm) e Lycosidae (10 mm); nos períodos que apresentaram indivíduos com menor tamanho corporal (como "antes"), houve a influência de pequenas aranhas como Theridion sp. e outras das famílias Linyphiidae e Thomisidae; neste período não foram encontradas aranhas com mais do que $6 \mathrm{~mm}$.
O período "depois", que após a colheita do arroz ainda manteve algumas aranhas de maior porte como $A$. veniliae (7 - $8 \mathrm{~mm})$ e Tetragnatha aff.jaculator $(6,5 \mathrm{~mm})$, novamente ocorre diminuição do tamanho médio devido a ocorrência de alguns pequenos Linyphiidae (Sphecozone modica Millidge, 1991, Sphecozone sp. 2) e de espécies exclusivas como Euophrys aff. saitiformis e Ashtabula sp. 2. Young \& EdwARds (1990) revelam que $1 / 3$ das 42 espécies registradas medem menos que $4 \mathrm{~mm}$ de tamanho corporal; provavelmente estas pequenas aranhas predam pragas como trips, afídios e imaturos de Heteroptera e Lepidoptera. Este mesmo fato deve ocorrer na lavoura de arroz deste estudo, pois o tamanho médio dos adultos foi apenas um pouco maior $(4,61 \mathrm{~mm}$, durante a lavoura) que o registrado pelos autores para agroecossistemas nos Estados Unidos.

Considerações finais. A carência de estudos da araneofauna em lavouras de arroz irrigado no Brasil dificulta a comparação e discussão dos resultados taxonômicos e dos padrões encontrados nesta pesquisa. Este trabalho demonstra que, mesmo em ambientes com intensas modificações antrópicas como os agroecossistemas e sucessivos distúrbios à estrutura do habitat, a diversidade biológica é vasta e ainda desconhecida. A capacidade das aranhas de enfrentar estes distúrbios e colonizar constantemente ambientes perturbados leva este táxon a ter importância ecológica elevada nos agroecossistemas. Dados complementares sobre fontes de dispersores para as áreas de arroz aqui estudadas deverão elucidar os mecanismos de montagem das comunidades (sensu DiAmond, 1975). Pesquisas realizadas ao longo de vários anos em outros países (BARrion, 1999; Bambaradeniya et al., 2004) têm demonstrado que a riqueza de espécies em plantações de arroz é mais alta do que o esperado. Conforme STINNER et al. (1997), a análise da biodiversidade é um princípio fundamental na agricultura sustentável e este exame é 
imprescindível para os princípios do manejo dos sistemas agriculturáveis. Há a necessidade de que se desenvolvam estudos a longo prazo que contribuam para o conhecimento do impacto dos agroecossistemas nas populações de aranhas que habitam as áreas circunvizinhas às culturas e de pesquisas que venham complementar a lista de aranhas que estão associadas às lavouras de arroz e, até mesmo, a descoberta de espécies que venham contribuir como agentes em programas de controle biológico de pragas (IPM) para a orizocultura que predomina em grandes extensões no Rio Grande do Sul e no Brasil.

Agradecimentos. À curadora da coleção aracnológica $\mathrm{MCN}$, Erica H. Buckup, pelo acesso ao material e à bibliografia. Ao Dr. Antonio D. Brescovit (Instituto Butantan) e todos os pesquisadores que enviaram bibliografia. Aos técnicos e pesquisadores da EEA, IRGA, Cachoeirinha, RS, em especial ao Eng. Agrônomo Jaime Vargas de Oliveira pelo apoio. À Maria Aparecida de L. Marques (MCN) e ao Dr. Arno A. Lise (PUCRS) pela determinação de alguns Theridiidae e Thomisidae, respectivamente. À direção do MCN pelo uso das dependências e equipamentos. Ao CNPq e à CAPES pela concessão das bolsas a E. N. L. Rodrigues e M. Mendonça Jr., respectivamente.

\section{REFERÊNCIAS BIBLIOGRÁFICAS}

Aguilar F. P. G. 1989. Las arañas como controladoras de plagas insectiles en la agricultura peruana. Revista Peruana de Entomologia 31:1-8.

Altieri, M. A. \& Nicholls, C. I. 2003. Biodiversity and pest management in agroecosystems. 2.ed. New York, The Haworh Press. 236p.

Ambalagan, G. \& Narayanasamy, P. 1999. Population fluctuation of spiders in the rice ecosystem of Tamil Nadu. Entomol 24(1):91-95.

Bambaradeniya, C. N. B. \& Edirisinghe, J. P. 2001. The ecological role of spiders in the rice fields of Sri Lanka. Biodiversity 2(4):3-10.

Bambaradeniya, C. N. B.; Edirisinghe, J.P.; de Silva, D. N.; Gunatilleke, C. V. S; Ranawana, K. B. \& Wijekoon, S. 2004. Biodiversity associated with an irrigated rice agro-ecosystem in Sri Lanka. Biodiversity and Conservation 13:1715-1753.

BARRION, A. T. 1999. Guild structure, diversity and relative abundance of spiders in selected non-rice habitats and irrigated rice fields in San Juan, Batangas, Philippines. Philippine Entomologist 13(2):129-157.

Bastidas, H.; Pantoja, A.; Zuluaga, I. \& Murillo, A. 1993. Colombian ricefield spiders. International Rice Research Notes 18(2):32-33

Bastidas, H.; Pantoja, A.; Murillo, A.; Zuluaga, I. \& Duque, M. C. 1994. Reconocimiento, flutuación y pruebas de consumo de presas por arañas en cultivos de arroz, en el Valle del Cauca. Revista Colombiana de Entomologia 20(3):149-160.

Снао, A. 1984. Non-parametric estimation of the number of classes in a population. Scandinavian Journal of Statistics 11:265-270.

Coddington, J. A. \& Levi, H. W. 1991. Systematics and evolution of spiders (Araneae). Annual Review of Ecology and Systematics 22:565-592.

Colwell, R. K. 2005. EstimateS: Statistical estimation of species richness and shared species from samples. Version 7.5. Disponível em: <http://viveroy.eeb.uconn.edu/ EstimateS>

Corseuil, E.; Brescovit, A. D. \& Heineck, M. A. 1994a. Aranhas associadas à cultura da soja em Eldorado do Sul, Rio Grande do Sul. Biociências 2(1):95-105.

Corseuil, E.; Paula, M. C. Z. \& Brescovit, A. D. 1994b. Aranhas associadas a uma lavoura de arroz irrigado no município de Itaqui, Rio Grande do Sul. Biociências 2(2):49-56.

Diamond, J. M. 1975. Assembly of species communities. In: Diamond, J. M. \& Cody, M. L. eds. Ecology and Evolution of Communities. Cambridge, Harvard University Press. p.342-444.
Dumas, B. A.; Boyer, W. P. \& Whitcomb, W. 1964. Effect of various factors on surveys of predaceous insects in soybeans. Journal of the Kansas Entomological Society 37:192-201.

Foelix, R. F. 1982. Biology of spiders. Cambridge, Massachusetts, Harvard University Press. 306p.

Greenstone, M. H. \& Sunderland, K. D. 1999. Why a symposium on spiders in agroecosystems now? The Journal of Arachnology 27:267-269.

GunNARsson, B. 1990. Vegetation structure and the abundance and size distribution of spruce-living spiders. Journal of Animal Ecology 59:743-752.

Hammer, O. \& Harper, D. A. T. 2005. PASt: Paleontological Statistics, version 1.34. Disponível em: <http://folk.uio.no/ ohammer/past>

HöFer, H. \& BRESCovit, A. D. 2001. Species and guild structure of a Neotropical spider assemblage (Araneae) from Reserva Ducke, Amazonas, Brazil. Andrias 15:99-119.

Horner, N. V. 1975. Annual aerial dispersal of jumping spiders in Oklahoma. The Journal of Arachnology 2:101-105.

IRGA. 2004. Estação experimental do arroz: 65 anos gerando tecnologia. Lavoura Arrozeira 52(435):32-39.

LeE, J. H. \& Kim, S. T. 2001. Use of spiders as natural enemies to control rice pest in Korea. Disponível em: <www.agnet.org/library/article/eb501.html\#eb501f1>. Acesso em: 16.04 .2005

Levi, H. W. 1988. The neotropical orb-weaving spiders of the genus Alpaida (Araneae: Araneidae). Bulletin of the Museum of Comparative Zoology 151(7):365-487.

Lewinsohn, T. M. \& Prado, P. I. 2002. Biodiversidade brasileira: síntese do estado atual do conhecimento São Paulo, Contexto Acadêmica. 176p.

Liljerthröm, G.; Minervino, E.; Castro, D. \& Gonzalez, A. 2002 La comunidad de aranãs del cultivo de soja en la Provincia de Buenos Aires, Argentina. Neotropical Entomology 31(2): 197-210.

Magurran, A. E. 1988. Ecological diversity and its measurement. New Jersey, Princeton University Press. $179 \mathrm{p}$.

Medina, A. C. 1994. Las arañas: controladores naturales de insectos em el cultivo de arroz em Norte de Santander. Revista Colombiana de Entomologia 20(3):179-186.

MuratA, K. 1995. The interaction between spiders and prey insects under sustainable cultivation - influence of paddy field managements on the densities of spiders and their prey insects. Acta Arachnologica 44(1):83-96.

NunEz, G. I. \& Ballinas, J. A. G. 1998. Diversidade de tres familias de arañas tejedoras (Araneae: Araneidae, Tetragnathidae, Theridiidae) en cafetales del Soconusco, Chiapas, Mexico. Folia Entomologica Mexicana 102:11-20.

Nyffeler, M. \& Benz, G. 1987. Spiders in natural pest control: a review. Journal of Applied Entomology 103:321-329.

Oraze, M. J.; Grigarick, A. A.; Lynch, J. H. \& Smith, K. A. 1988. Spider fauna of flooded rice fields in northen California. The Journal of Arachnology 16:331-337.

Patel, M. L.; Patel, K. G. \& Desai, H. R. 2004. Spider fauna of rice of South Gujarat. Insect Environment 10(3):119-121.

PekÁr, S. \& Kocoukek, F. 2004. Spiders (Araneae) in the biological and integrated pest management of apple in the Czech Republic. JEN 128(8):561-566.

Platnick, N. I. 2008. The World Spider Catalog, Version 9.0. New York. Disponível em: <http://research.amnh.org/ entomology/spiders/catalog/index.html>. Acesso em: 01.09.2007.

Pommeresche, R. 2004. Surface-active spiders (Araneae) in ley and field margins. Norwegian Journal of Entomology 51:57-66.

Rypstra, A. L.; Carter, P. E.; Balfourd, R. A. \& Marshall, S. D. 1999. Architectural features of agricultural habitats and their impact on the spiders inhabitants. The Journal of Arachnology 27:371-377

Stinner, D. H.; Stinner, B. R. \& Martsolf, E. 1997. Biodiversity as an organising principle in agroecosystem management: case studies of holistic resource management practitioners in the USA. Agricultural Ecosystems and Environment 62:199-213.

Teixeira, M. B.; Coura-Neto, A. B.; Pastore, U. \& Rangel, A. L. R 1986. Vegetação; as regiões fitoecológicas, sua natureza, seus 
recursos econômicos; estudo fitogeográfico. In: Levantamento de recursos naturais. Instituto Brasileiro de Geografia e Estatística, Rio de Janeiro, v.33, p.541-632.

ThorbeK, P.; Sunderland, K. D. \& Topping, C. J. 2004. Reproductive biology of agrobiont linyphiid spiders in relation to habitat, season and biocontrol potential. Biological Control 30: $193-202$.

Turnbull, A. L. 1973. Ecology of the true spiders (Araneomorphae). Annual Review of Entomology 18:305-348.

Uetz, G. W.; HalaJ, J. \& CADY A. B. 1999. Guild structure of spiders in major grops. The Journal of Arachnology 27:270-280.

van Wingerden, W. K. R. E. \& Vugts, H. F. 1974. Factors influencing aeronautic behaviour of spiders. The Bulletin of British Arachnological Society 3(1):6-10.

ViJaykumar, P. B. V. 2004. Spider fauna of paddy ecosystem in selected areas of Tungabhadra Project in Karnataka. Journal of Agricultural Sciences 17(3):584-585.

Wise, D. H. 1993. Spiders in ecological webs. Cambridge, Cambridge University Press. 328p.

Woods, M. W. \& Harrel, R. C. 1976. Spider populations of a southeast Texas rice fields. Southwestern Naturalist 21:37-48.

YounG, O. P. \& Edwards, G. B. 1990. Spiders in United States field crops and their potential effect on crop pests. The Journal of Arachnology 18(1):1-27.

Recebido em janeiro de 2006. Aceito em julho de 2008. ISSN 0073-4721

Artigo disponível em: www.scielo.br/isz 